\title{
Miniature lightweight X-ray optics (MiXO) for surface elemental composition mapping of asteroids and comets
}

\author{
Jaesub Hong ${ }^{1 *}$, Suzanne Romaine ${ }^{2}$ and The MiXO team
}

\begin{abstract}
The compositions of diverse planetary bodies are of fundamental interest to planetary science, providing clues to the formation and evolutionary history of the target bodies and the solar system as a whole. Utilizing the X-ray fluorescence unique to each atomic element, $X$-ray imaging spectroscopy is a powerful diagnostic tool of the chemical and mineralogical compositions of diverse planetary bodies. Until now the mass and volume of focusing X-ray optics have been too large for resource-limited in situ missions, so near-target $X$-ray observations of planetary bodies have been limited to simple collimator-type X-ray instruments. We introduce a new Miniature lightweight Wolter-I focusing X-ray Optics (MiXO) using metal-ceramic hybrid X-ray mirrors based on electroformed nickel replication and plasma thermal spray processes. MiXO can enable compact, powerful imaging $X$-ray telescopes suitable for future planetary missions. We illustrate the need for focusing X-ray optics in observing relatively small planetary bodies such as asteroids and comet nuclei. We present a few example configurations of MiXO telescopes and demonstrate their superior performance in comparison to an alternative approach, micro-pore optics, which is being employed for the first planetary focusing X-ray telescope, the Mercury Imaging X-ray Spectrometer-T onboard Bepicolumbo. X-ray imaging spectroscopy using MiXO will open a large new discovery space in planetary science and will greatly enhance our understanding of the nature and origin of diverse planetary bodies.
\end{abstract}

Keywords: X-ray fluorescence, Elemental abundance, X-ray imaging

\section{Background}

ROSAT, the Chandra X-ray Observatory and other X-ray observatories have revealed that most planetary bodies in our solar system emit X-rays via various physical processes including $\mathrm{X}$-ray fluorescence (XRF) and charge exchange with plasma. In XRF, the observed energies are unique to the atomic levels of each element. This property of XRF enables a powerful way to probe the surface distribution of elemental composition in the emitting bodies. Figure 1 shows a simulated X-ray spectrum from an asteroid of $\mathrm{C} 1$ chondrite composition located at $1 \mathrm{AU}$ during a typical quiet sun state. X-ray emission from the asteroid contains numerous XRF emission lines tied to various atomic elements (Nittler et al. 2004). Figure 1 also

\footnotetext{
*Correspondence: jhong@cfa.harvard.edu

${ }^{1}$ Harvard University, 60 Garden Street, Cambridge, MA 02138, USA Full list of author information is available at the end of the article
}

shows how the relative abundance of $\mathrm{Mg} / \mathrm{Si}$ vs. S/Si can be used to identify matching meteorite specimen types.

While Earth-orbiting X-ray observatories are capable of high angular resolution X-ray imaging (e.g. sub-arcsec angular resolution for Chandra), the large distance to relatively $\mathrm{X}$-ray faint rocky airless bodies severely limits the photon statistics and often makes it difficult to identify interesting surface features or substructures of X-ray emission. For instance, the observed X-ray flux of the Crab nebula, one of the brightest X-ray sources in the sky, is a few photons $\mathrm{cm}^{-2} \mathrm{~s}^{-1} \mathrm{keV}^{-1}$ at around $1 \mathrm{keV}$. This is comparable to the XRF flux from an asteroid located at 1 AU from the Sun observed by a telescope with an FoV of $\gtrsim 10 \mathrm{deg} \times 10 \mathrm{deg}$ at the surface. Due to the Lambertian reflectance, the XRF flux observed by such a telescope will be more or less the same regardless of the distance as long as the FoV is fully occupied by the emitting

\section{至 Springer}

(C) 2016 Hong and Romaine. This article is distributed under the terms of the Creative Commons Attribution 4.0 International License (http://creativecommons.org/licenses/by/4.0/), which permits unrestricted use, distribution, and reproduction in any medium, provided you give appropriate credit to the original author(s) and the source, provide a link to the Creative Commons license, and indicate if changes were made. 

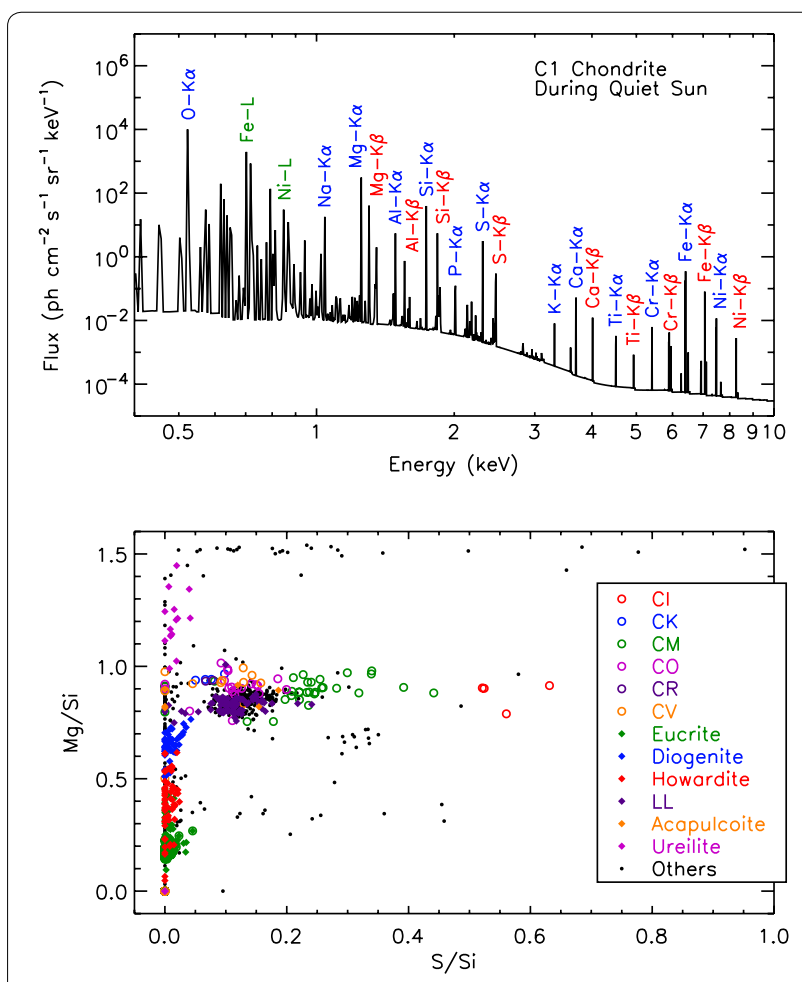

Fig. 1 (Top) Simulated X-ray fluorescence spectrum of an asteroid of C1 chondrite composition at $1 \mathrm{AU}$ during the quiet sun state illustrating diverse elemental composition. (Bottom) Abundance ratios (Mg/Si vs. S/Si) as an identifier of a wide range of meteorite specimen types (Nittler et al. 2004)

surface. On the other hand, if the asteroid of $\sim 1 \mathrm{~km}$ size is observed at a distance of roughly $1000 \mathrm{~km}$, only a tiny fraction of the XRF flux $\left(10^{-5}\right)$ will reach the telescope since the emission of the XRF is more or less isotropic.

$\mathrm{X}$-ray optics in the past are massive, so X-ray instruments flown on near-target missions in the past have foregone true imaging capabilities. Instead, they had to employ simple collimator-type instruments as in the X-ray spectrometers (XRS) on NEAR-Shoemaker (Trombka et al. 2000), Hayabusa (Okada et al. 2006) or Messenger (Nittler et al. 2011). This is largely because a large portion of the resources in a typical planetary mission often has to be reserved for the spacecraft to make possible the long journey to the target and the remaining resources are shared by a collection of onboard instruments required to unveil the mysteries of the targets. Hence the resources allowed for each instrument are limited, and conventional X-ray focusing optics used in the past are simply too large and too heavy to be a part of planetary missions.

Despite the lack of imaging capability, X-ray observations of the targets have been proven to provide unique insights. For instance, XRS observations of 433 Eros by
NEAR-Shoemaker revealed that the elemental composition of the asteroid from the X-ray spectra is different from the asteroid classification acquired by the optical and infrared spectra (Trombka et al. 2000). It is suspected that ongoing space weathering alters the surface of the asteroids up to a few $\mu \mathrm{m}$ depth, which modifies the optical and infrared spectra, whereas the XRF probes more than $20 \mu \mathrm{m}$ below the surface and can reveal the true, unweathered, composition (Binzel et al. 2010). In the case of Itokawa, definitive evidence of space weathering on the asteroid was later found in the returned sample (Noguchi et al. 2011, 2014).

From recent images of Comet 67P/ Churyumov-Gerasimenko taken by NAVCAM $\left(\sim 18^{\prime \prime}\right.$ resolution) on the Rosetta mission, one can imagine exciting potential of future X-ray observations of asteroids and comets when $\mathrm{X}$-ray spectroscopy is combined with high-resolution imaging. The optical image of Comet 67P shows diverse terrain and volatile activity (Fig. 2). In particular, the high ratio of deuterium in the water vapor of the jets questions our understanding of the origin of the water on Earth (Altwegg et al. 2015). While soft X-ray observations (0.1$15 \mathrm{keV}$ ) are insensitive to isotope variation, $\mathrm{X}$-ray imaging spectroscopy can separate the elemental composition of gaseous jets from the comet's surface as well as the

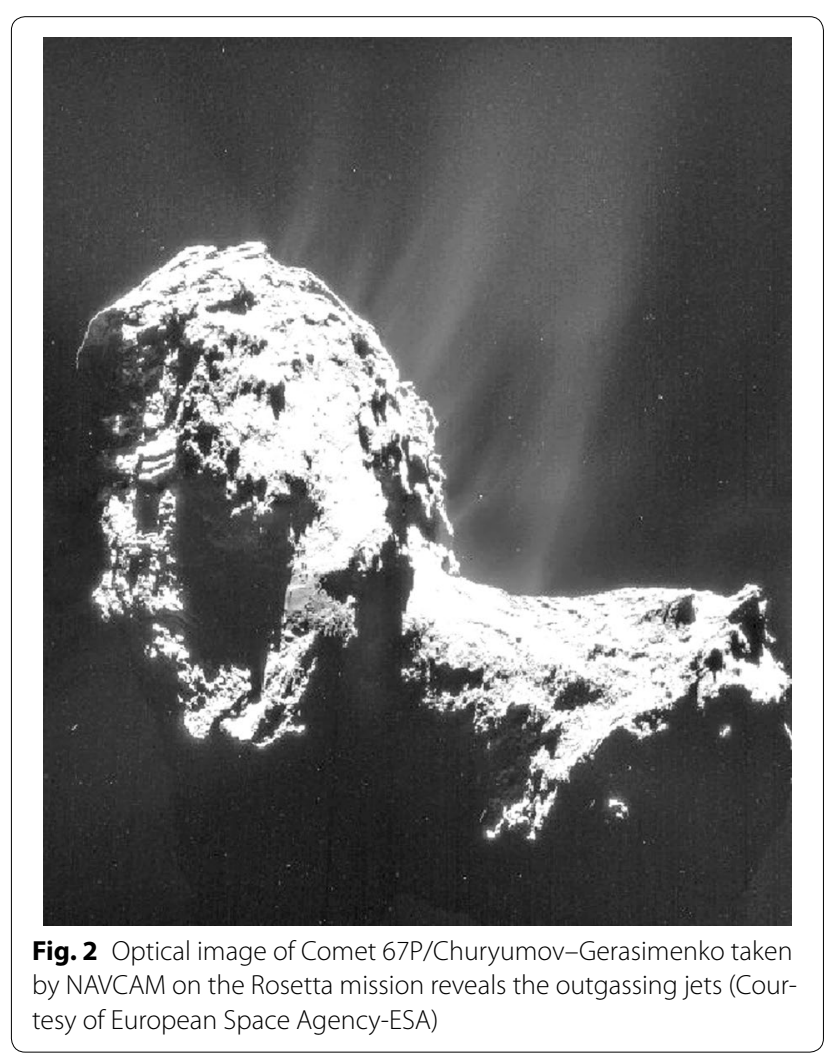


compositional variation of the surface due to impacts and volatile activities in the past.

As the need for sample retrieval of various planetary bodies rises for in-depth analysis in attempt to unambiguously unveil their nature, origin and evolutionary history, many future sample return planetary missions are being developed. The retrievable size of samples, however, is very small: e.g. sub-gram in Hayabusa 2 (Tachibana et al. 2013), less than $1 \mathrm{~kg}$ in OSIRIS-REx (Lauretta and Team 2012). Therefore, X-ray imaging spectroscopy onboard future near-target missions becomes an indispensable tool in acquiring a global context of the atomic composition for the returned samples as well as assisting the sample site selection during the mission.

In "Advantages of focusing X-ray optics" section, we review the major challenges encountered in near-target $\mathrm{X}$-ray observations using collimator instruments and illustrate how focusing X-ray optics can overcome these challenges and enhance a chance of success and new discoveries especially in observing relatively small bodies such as asteroids and comet nuclei. In "Advances of X-ray optics: electroformed Nickel replication with thermal plasma spray" section, we briefly review the advances of Wolter-I X-ray optics and introduce our new approach to build compact, lightweight X-ray optics by merging Electroformed Nickel Replication (ENR) and Plasma Thermal Spray (PTS) processes. In "Example MiXO configurations and performance" section, we show example MiXO configurations suitable for planetary missions and estimate the performances in comparison to an alternative approach, micro-pore optics (MPO).

\section{Advantages of focusing X-ray optics}

$\mathrm{X}$-ray observations in near-target missions in the past have been challenging. Some of the challenges originate from incomplete prelaunch ground calibration of the instruments and inadequate onboard calibration system (usually due to tight assembly schedule and budget constraints). As a result, the data analysis was complex and often led to somewhat ambiguous interpretation. On the other hand, a cause of many challenges in the past planetary X-ray observation lies in the very nature of nonfocusing optics of collimator instruments as described below.

REgolith X-ray Imaging Spectrometer (REXIS) onboard OSIRIS-REx, which is scheduled to launch in 2016 to collect samples from Bennu, was designed to take into account lessons learned from the previous missions (Inamdar et al. 2014). For instance, REXIS will be equipped with one of the most comprehensive onboard calibration systems in space X-ray instruments: a set of ${ }^{55}$ Fe radioactive sources are strategically mounted to monitor any potential drifts or changes of spectral gain and resolution of every node of four X-ray CCDs onboard. In addition, the team plans to conduct a series of extensive ground and flight calibrations to understand the detector response and to track any variation during the observation.

REXIS utilizes coded-aperture imaging to identify $\sim 50 \mathrm{~m}$ scale surface variation of the elemental composition of Bennu. The addition of imaging capability is the first of its kind (coded-aperture imaging) in planetary X-ray observations (see below about the Bepicolumbo mission). Coded-aperture imaging is basically shadowgram imaging using a mask coded with a pattern of open and opaque elements in front of the detector. It is a novel technique to enable imaging without focusing optics, and thus allows a relatively compact, simple telescope design (REXIS fits in an envelope of $\sim 10 \mathrm{~cm} \times 10 \mathrm{~cm} \times 20 \mathrm{~cm}$ ). However, coded-aperture imaging still operates under the basic principle of collimator instruments, facing the same challenges. Thus, its imaging sensitivity is relatively low compared to focusing instruments and it has been mainly employed to identify point sources in astrophysics in the past.

When observing large planetary bodies such as the Moon, a simple collimator-based X-ray instrument can efficiently collect the X-rays from the surface and identify spatial variations as the instrument orbits around the target. The large gravity and the spherical geometry of the target with a small eccentricity allow stable circular orbits very close to the surface (Fig. 3a). In addition, the field of view (FoV) of the instrument is fully occupied by the target, so that no external background can contaminate the data.

In the case of relatively small planetary bodies such as asteroids and comet nuclei, the small gravity and the irregular shape of the target often limit stable orbital configurations near the target (Fig. 3b, c). To collect faint $\mathrm{X}$-ray emission from the target, the FoV of the instrument is usually designed to fill the target, and thus the collimator-type instrument cannot efficiently identify the surface feature of small scales. At the same time, the exposure to the background sky in the FoV should be limited because the X-ray sky is not dark, but it shines with many diffuse and point X-ray sources, often brighter than the target itself. For instance, our Galactic plane glows brightly in $\mathrm{X}$-ray, which is known as Galactic ridge X-ray emission from the early days of the X-ray astronomy. The sky at high Galactic latitude is also filled with X-ray emission from unresolved Active Galactic Nuclei (Fig. 4). These components contribute to bright Cosmic X-ray background $(\mathrm{CXB})$, which can often dominate $\mathrm{X}$-ray emission from the target in the collimator-type instruments when their FoV is exposed to the background sky. The continuum component of the CXB is about $10 \mathrm{ph} \mathrm{cm}^{-2} \mathrm{~s}^{-1} \mathrm{sr}^{-1}$ 

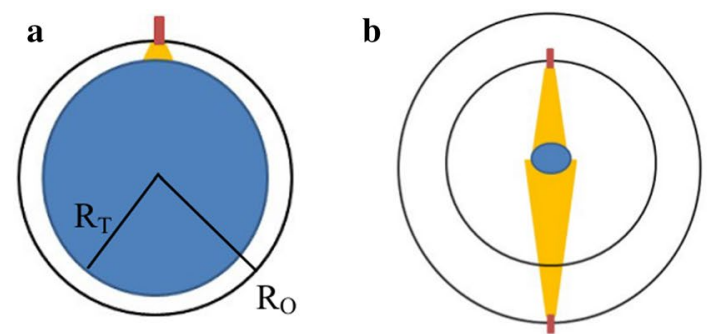

c

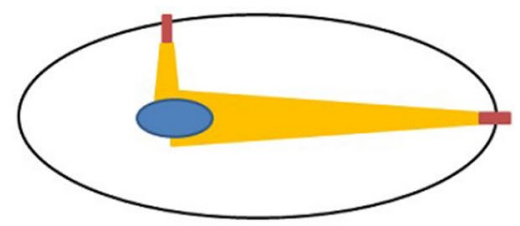

Fig. 3 a For large spherical planetary bodies (radius $R_{T}>\sim 1000 \mathrm{~km}$ ), observation can be conducted at a close stable orbit $\left(R_{0}\right)$ (i.e. $\left.R_{T} \sim R_{0}\right)$, where a simple collimator can be sufficient for identifying large surface variation. b, c For small targets $\left(R_{T}<\sim 100 \mathrm{~km}\right)$, low gravity or irregular shape prevents observation at a close steady orbit (i.e. $R_{T}<<R_{0}$ ), and the size of the imaging footprint on the surface can change dramatically over time or even in a single orbit (Hong and Romaine 2014)

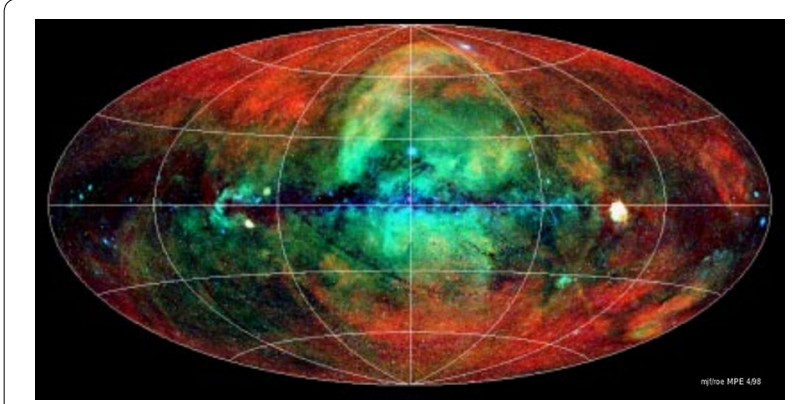

Fig. 4 All sky soft X-ray background image in Galactic coordinates by ROSAT/PSPC after removing bright point sources (red 0.1-0.4 keV, green $0.5-0.9$ keV, blue 0.9-2.0 keV, Courtesy of Max-Planck-Institut for extraterrestrische Physik)

$\mathrm{keV}^{-1}$ at $1 \mathrm{keV}$, which is about $100-1000$ times brighter than the example in Fig. 1.

$\mathrm{X}$-ray instruments in space also experience additional internal X-ray background which occurs due to interactions between cosmic-ray particles and the instruments. The internal background can contain XRF lines originated from the instrument, which can complicate the elemental identification of the target. The sensitivity of a typical collimator instrument is proportional to a square root of the detector area (instead of being linearly proportional to the detector area) because the internal background increases as the detector area increases. On the other hand, focusing telescopes allow a small focal plane with a large effective area, so that the internal background can be 10- to 100-fold smaller than the collimator instruments of similar collecting area.

Due to high CXB (and the large internal background), observations with collimator instruments are susceptible to changes in observing configurations (e.g. changes in pointing directions or observing distances), which can severely narrow the observing windows. Focusing telescopes, on the other hand, can start meaningful observations much farther out (e.g. during early phases of approach and debris scouting) by simply resolving and removing most of both external and internal backgrounds.

Figure 5 compares relative minimum exposures for XRF detection from an asteroid by well-calibrated XRS of NEAR-Shoemaker and Hayabusa with an example MiXO telescope (MiXO-70B, see "Example MiXO configurations and performance" section) as a function of standoff distance or orbit radius $\left(R_{\mathrm{O}}\right)$ normalized to the target size (radius, $R_{\mathrm{T}}$ ). It also shows a scaled example

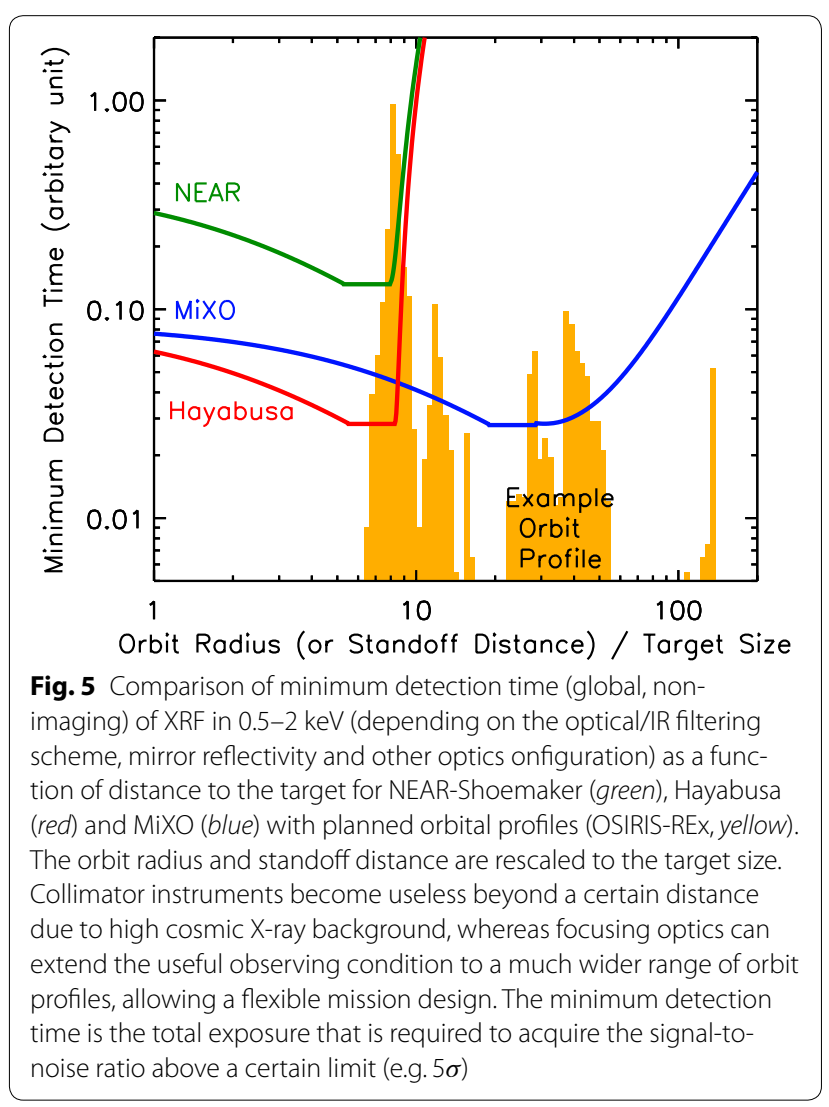


orbital/approach profile from OSIRIS-REx (the orange histogram). In situ or near-target experiments studying small asteroids $\left(R_{\mathrm{O}} \lesssim 100 \mathrm{~km}\right)$ often cannot stay in a stable orbit at close proximity (i.e. $R_{\mathrm{O}} / R_{\mathrm{T}}>>1$ ) due to low gravity, debris, or an irregular asteroid shape. In addition, the multiple stages of approach to the target make the overall orbital profiles to spread over a wide range of distances to the target.

Imaging by focusing X-ray optics enables a more forgiving orbit configuration for observation, and focusing telescopes can continue to accumulate useful data throughout the mission $\left(R_{\mathrm{T}} / R_{\mathrm{O}} \lesssim 300\right.$ for global measurements, $R_{\mathrm{T}} / R_{\mathrm{O}} \lesssim 100$ for mapping). As shown in Fig. 5 , the MiXO telescope would have completed the same measurements as NEAR-Shoemaker or Hayabusa even before they reached their observing distance. A longer observing period enabled by MiXO also enhances a chance of success and new discoveries, since detection of hard $\mathrm{X}$-rays from heavy elements often relies on relatively rare strong solar flares. In addition to the global measurement, with MiXO, the accumulated data over $\sim 2$ months (at $1 \mathrm{AU}$ ) during the quiet sun state alone will be sufficient for detection of $\sim 1 \%$ spatial variation for major elements (e.g. O-K, Fe-L) over the entire surface.

The advantages of focusing optics extend to observations of large planetary bodies as well. For instance, at Mercury, the high thermal loads force spacecraft to be at highly elliptical orbits, limiting the observing duty cycle of collimator instruments. With focusing optics less susceptible to changes in observing conditions, the observing duty cycle can be maintained high. In the case of the Jovian system, high radiation environment $(1 \mathrm{krad}$ per an orbit, depending on shielding, etc.) severely limits the detector and shield size, and thus, the focusing optics is required to achieve large light-collection power with a small detector.

\section{Advances of X-ray optics: electroformed Nickel replication with thermal plasma spray}

While the scientific motivation and the advantages of $\mathrm{X}$-ray focusing optics have been known, it has been impractical to implement conventional focusing X-ray telescopes in near-target planetary missions. As aforementioned, typical planetary missions are a multiinstrument discipline by nature, which is necessary but also limits the power, mass, volume and other resources allocated for each instrument. On the other hand, conventional X-ray optics tends to be heavy and large. This is because of relatively low areal density (i.e. relatively small collecting area per unit mass) of X-ray mirrors. $\mathrm{X}$-ray optics relies on grazing incidence for efficient X-ray reflection, and a set of barrel shape mirrors are stacked together in the Wolter-I configuration to establish needed effective area. For instance, the outer X-ray mirror of Chandra is about $1.4 \mathrm{~m}$ diameter by $1 \mathrm{~m}$ long with $10 \mathrm{~m}$ focal length, and weighs about $1500 \mathrm{~kg}$.

Figure 6 illustrates the advances of X-ray optics in weight reduction over the years while maintaining high angular resolution $\left(<15^{\prime \prime}-30^{\prime \prime}\right)$ and improving collecting power. In Chandra, high-resolution mirrors were made from directly grinding and polishing glass ceramic (zerodur), which enabled sub-arcsec angular resolution (Fig. 6a). Electroformed Nickel Replication (ENR) process used to build X-ray mirrors in XMM-Newton (Fig. 6b) utilizes relatively thin Ni shell $\left(1 \mathrm{~mm}, 8.9 \mathrm{~g} / \mathrm{cm}^{3}\right)$ as a substrate for the support of mirror figure and shape. The thin Ni shell enables reduction of the overall mass by a factor of more than five compared to X-ray mirrors in Chandra and allows larger light collecting area under the same packaging volume. In ENR, the mirror figure and angular resolution are determined by the surface quality of mandrel, and $10^{\prime \prime}-15^{\prime \prime}$ can be achieved routinely. The Astronomical Roentgen Telescope (ART) (Gubarev

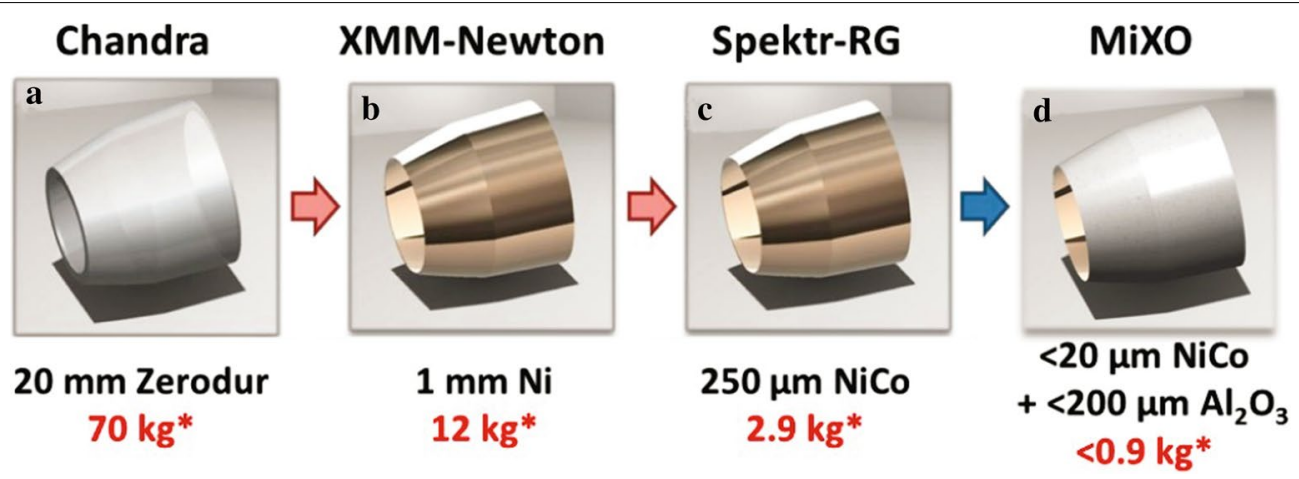

Fig. 6 Advances of X-ray optics. a A ground and polished glass substrate, $\mathbf{b}$ electroformed Ni shell, c thin electroformed NiCo shell, and $\mathbf{d}$ a metalceramic hybrid shell. The weights (asterisk) are for a $70 \mathrm{~cm}$ dia., $60-\mathrm{cm}$-long mirror shell. For small mirrors ( $\sim 5 \mathrm{~cm}$ dia.) suitable for planetary science, a single shell would weigh $\sim 10 \mathrm{~g}$ or less 
et al. 2012) onboard Spektr-RG, which is scheduled to launch in 2016, employs an NiCo alloy ( $250 \mu$ m thick) as a substrate instead of Ni under the ENR process, further reducing the mirror mass (Fig. 6c).

Recently we have begun to develop a new approach, where we combine the Plasma Thermal Spray (PTS) technology and the ENR process to form metal-ceramic hybrid X-ray mirrors (Romaine et al. 2014). In the hybrid X-ray mirrors, the lightweight ceramic layer provides the stiffness needed to maintain the overall figure of X-ray optics, while the NiCo layer maintains the low surface roughness necessary for X-ray reflection. By replacing the majority of the NiCo layer $\left(\sim 8.9 \mathrm{~g} / \mathrm{cm}^{3}\right)$ with the lightweight alumina $\left(\mathrm{Al}_{2} \mathrm{O}_{3}, 2.3-2.9 \mathrm{~g} / \mathrm{cm}^{3}\right)$, we can further reduce the mirror mass by another factor of 30 compared to normal ENR mirrors, depending on the thickness of mirrors. Figure 7 shows example modules: a conical mirror mandrel, a $\mathrm{NiCo}$ shell, and two NiCo ceramic hybrid shells from left to right. The modules in Fig. 7 is single bounce, but ENR process can form a mirror with two bounces in one package, simplifying the alignment structure without introducing any degradation in angular resolution (e.g. see Gubarev et al. 2012; Romaine et al. 2014). The hybrid shells in Fig. 7, which demonstrate a proof of concept, are made of a $300-\mu$ $\mathrm{m}$ ceramic layer with $100 \mu \mathrm{m}$ of an NiCo layer. Given the properties of $\mathrm{NiCo}$ and ceramic layer, we expect that sub$20 \mu \mathrm{m} \mathrm{NiCo}+200 \mu \mathrm{m}$ ceramic X-ray mirror shell can be achieved for relatively small shells $(2-20 \mathrm{~cm}$ diameter).

\section{Example MiXO configurations and performance}

MiXO made of metal-ceramic hybrid X-ray mirrors shows a tremendous promise in enabling compact X-ray telescopes suitable for various types of planetary missions, which can open a new era of sensitive X-ray imaging spectroscopy in planetary science. Until now, micro-pore optics

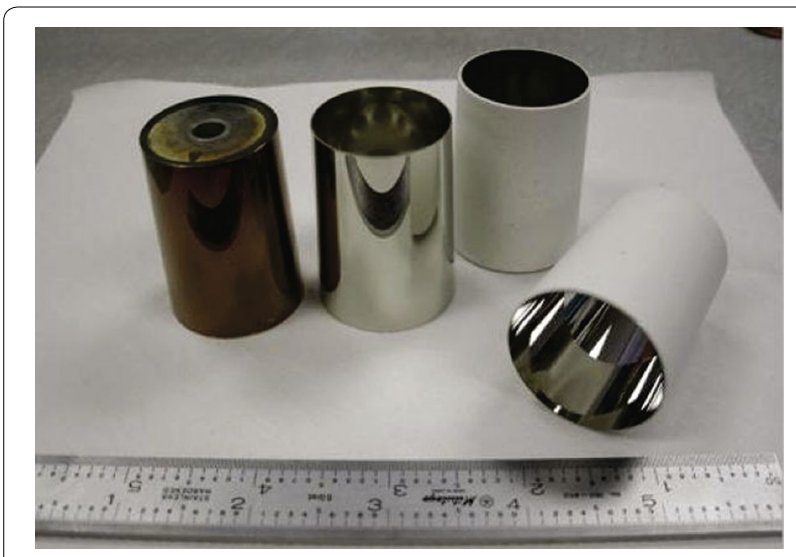

Fig. 7 A test single-bounce conical mandrel, an NiCo shell, two NiCo ceramic hybrid shells from left to right. The width of the image spans about $14 \mathrm{~cm}$
(MPO) was considered the only approach that can enable compact Wolter-I X-ray optics in planetary missions. The Mercury Imaging X-ray Spectrometer-T (MIXS-T) on Bepicolombo employs the MPO and will be the first focusing X-ray telescope in planetary science missions (Fraser et al. 2010). In MPO of MIXS-T, the inner surface of small square pores or microchannels $(\sim 20 \mu \mathrm{m}$ pitch) in a 0.9 - to 2.2-mm-thick plate is coated with IR for X-ray reflection. Then, mosaics of segmented MPO plates are assembled to form a Wolter-I configuration. In this section, we estimate the performance of MiXO using a few example configurations in comparison with Bepicolombo/MIXS-T.

Figure 8 and Table 1 show two example configurations using the hybrid shells at $70 \mathrm{~cm}$ focal length: the baseline option (MiXO-70B) (left) and a wide field option

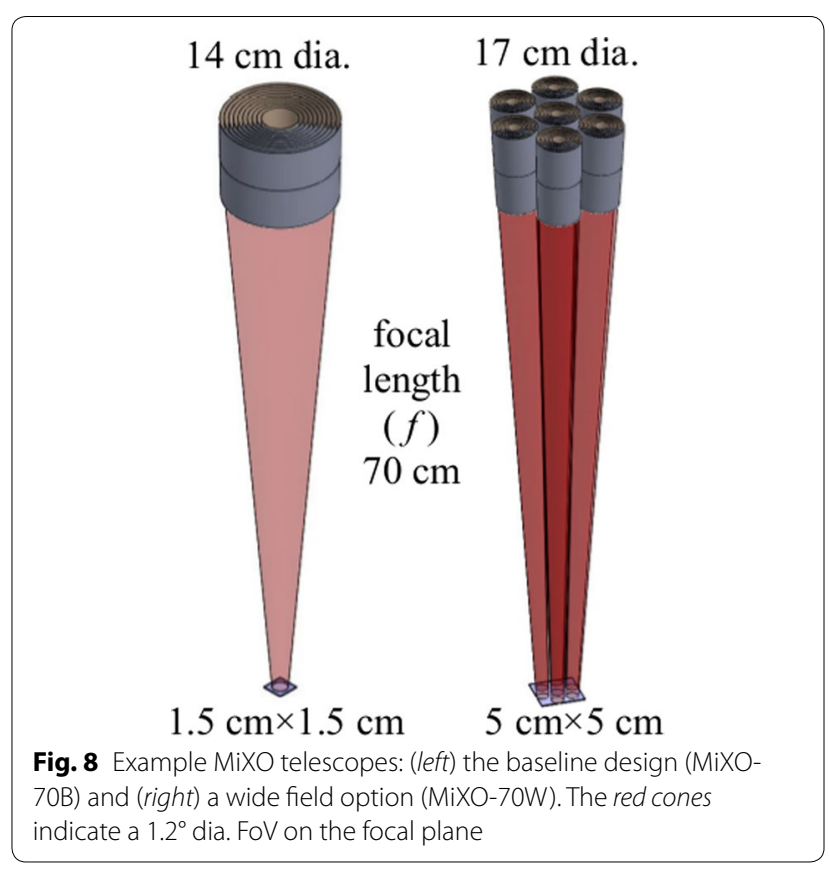

Table 1 Example MiXO telescopes

\begin{tabular}{lll}
\hline Parameters & MiXO-70B & MiXO-70W \\
\hline Focal length $(f)$ & $70 \mathrm{~cm}$ & $70 \mathrm{~cm}$ \\
No. of shells & 50 & $25 \times 7$ \\
Size (diameter) & $4-14 \mathrm{~cm}$ & $2-5.6 \mathrm{~cm}$ \\
& & $17 \mathrm{~cm}$ overall \\
Mass (g) & 920 & $210 \times 7$ \\
Detector $\left(\mathrm{cm}^{2}\right)$ & $1.5 \times 1.5$ & $5 \times 5$ \\
FoV (deg $\left.{ }^{2}\right)$ & 1 & 7 \\
Ang. resolution & $30^{\prime \prime}$ & $30^{\prime \prime}$ \\
Effective area & $65 \mathrm{~cm}^{2} @ 1 \mathrm{keV}$ & $6.4 \mathrm{~cm}^{2} @ 1 \mathrm{keV}$ \\
& $15 \mathrm{~cm}^{2} @ 4 \mathrm{keV}$ & $4.6 \mathrm{~cm}^{2} @ 4 \mathrm{keV}$ \\
Grazing angle $\left(^{\circ}\right)$ & $0.41-1.43$ & $0.20-0.51$ \\
\hline
\end{tabular}

Assume $10 \%$ reduction for alignment fixture 
(MiXO-70W) (right). Each shell in both the options consists of $20 \mu \mathrm{m} \mathrm{NiCo}+200 \mu \mathrm{m} \mathrm{Al} \mathrm{O}_{3}$ layers. It is $\sim 10$ $\mathrm{cm}$ long ( $\sim 5 \mathrm{~cm}$ for each bounce), and the gap between the adjacent shells is $500 \mu \mathrm{m}$. The optics alone weighs $\sim$ $0.9-1.5 \mathrm{~kg}$, and the support structure will add another $0.3-0.5 \mathrm{~kg}$ under a conservative estimate. The baseline design is scalable to a smaller or larger optics depending on available resources or mission objectives. For example, a bigger telescope similar to Bepicolombo/MIXS-T $(f \sim 100 \mathrm{~cm})$ or a smaller telescope similar to JUXTA $(f \sim 25 \mathrm{~cm}$ Ezoe et al. 2013) can be implemented. MiXO-70W is optimized for wide field monitoring with the combined FoV of $7 \mathrm{deg}^{2}$ and broad band coverage with relatively higher grasp at high energies due to small grazing angles of each module. The FoV of each module in MiXO-70W is independent of each other, so that the alignment between them is not critical.

The sensitivity or light collection power of an X-ray telescope is often described by the on-axis effective area. Since X-ray emission from planetary bodies is often diffuse by nature especially when observed nearby, another important parameter to describe the performance of X-ray telescopes is grasp, which is the product of the effective area and the FoV. Figure 9 compares the expected grasp of various MiXO optics with MIXS-T on Bepicolombo. We assume that each shell of MiXO here is coated with IR. With only $\sim 1 / 3$ of the packaging volume, MiXO-70B (\$) meets the performance of MIXS-T (\$); or MiXO-100B ( 1 in Fig. 9, the same baseline configuration as MiXO-70B but $f \sim 100 \mathrm{~cm}$ with an optics envelope of $20 \mathrm{~cm}$ dia.), whose package is similar to MIXS-T in size, outperforms MIXS-T by a factor of $\sim 3$. In the case of MiXO-70W ( $\downarrow)$, small grazing angles boost high energy response, enhancing a chance of the discovery of

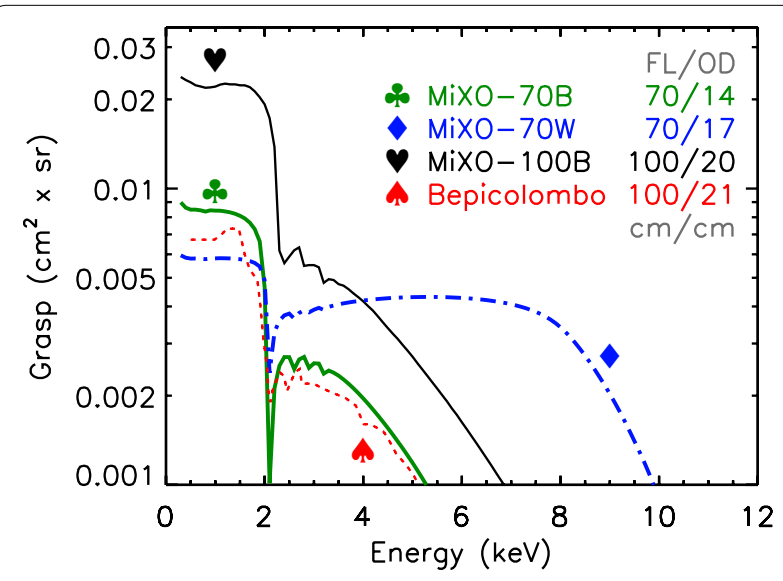

Fig. 9 Grasp of MiXO-70B, MiXO-70W and MiXO-100B optics with IR coating (10\% reduction for support structure, no surface or multilayer optimization) in comparison with MIXS-T on Bepicolombo (Fraser et al. 2010). (FL focal length, OD optics diameter) heavy elements. Since our approach enables additional optimizations such as polynomial profile of shell geometry for uniform response over the wide field (Conconi et al. 2010) or multilayer coating to enhance hard X-ray response (e.g. NuSTAR; Harrison et al. 2013), one can further improve the response of MiXO telescopes.

In addition to outperforming on throughput (both onaxis effective area and overall grasp) by a factor of three or more, our approach excels MPO in two other areas: first, MPO can focus X-rays effectively only below $2 \mathrm{keV}$ due to the difficulty in coating the pore surface on the $20-\mu \mathrm{m}$ scale. For instance, multilayer coating cannot be applied to small pores. On the other hand, MiXO-70W in Fig. 9 is designed for wide field hard $(>2 \mathrm{keV}) \mathrm{X}$-ray imaging and thus optimized for the detection and localization of heavy elements such as rare-earth elements.

Second, the resolution of MPO is limited to $2^{\prime}$ for lobster eye Angel optics and $5-8^{\prime}$ for Wolter-I optics. The additional degradation in resolution for Wolter-I optics comes from misalignment among segmented mirror facets in MPO. In fact, difficult alignment between segmented mirrors requires a complex support structure, which mostly erases its lightweight advantage relative to $\mathrm{MiXO}$. On the other hand, in MiXO, each shell contains both bounces, greatly simplifying alignment structure and mass, and a 15-30" resolution (10-16 $\times$ improvement relative to MPO) can be achieved routinely. Compared to MIXS-T, MiXO-70B, though a smaller telescope, improves the detection sensitivity ( $\propto$ PSF_area ${ }^{1 / 2}$ ) of small surface features by $>10 \times$ or extends the observing distance $\left(\propto\right.$ PSF_area ${ }^{1 / 4}$ ) by $>3 \times$ due to relatively lower background $(\propto$ PSF_area) of small PSFs enabled by high resolution $\left(<30^{\prime \prime}\right)$.

High angular resolution planetary X-ray imaging with sub $100 \mathrm{~cm}^{2}$ effective area will be normally flux limited during the quiet Sun phases. On the other hand, the full potential of the high angular resolution can be realized during strong solar X-ray flares which can generate higher solar X-ray flux by a few orders of magnitude. Given the angular resolution in MiXO that is comparable to NAVCAM on the Rosetta mission, a strong solar X-ray flare can enable an X-ray snapshot of the detailed atomic composition, which can be directly compared to the surface structure of comet nuclei and outgassing jets in an optical image.

\section{Summary}

We have introduced a new approach to build lightweight focusing X-ray optics with metal-ceramic hybrid X-ray mirrors through the ENR process and the PTS technology. Hybrid X-ray mirrors are lighter by almost two orders of magnitude relative to X-ray mirrors in Chan$d r a$. This enables compact lightweight $\mathrm{X}$-ray telescopes 
which can be mounted on resource-limited in-situ or near-target planetary missions.

We have illustrated the benefit of true imaging X-ray spectroscopy especially for observations of small planetary bodies such as asteroids and comet nuclei. With a few example configurations we have demonstrated efficient light-collection power $(3 \times$ improvement relative to an alternative approach using MPO), high angular resolution $(>10 \times$ improvement), high detection sensitivity $(>10 \times$ improvement) and wide energy band coverage (up to $10-15 \mathrm{keV}$ with multilayer coating) of MiXO. With $\mathrm{MiXO}$, we can open a new era of planetary X-ray imaging spectroscopy.

\section{Authors' contributions}

$\mathrm{JH}$ carried out design and modeling work for MiXO, investigated its feasibility and applications to various planetary missions, and drafted the manuscript. SR demonstrated the proof of concept of MiXO by fabricating and testing prototype MIXO modules. All authors read and approved the final manuscript.

\section{Author details}

${ }^{1}$ Harvard University, 60 Garden Street, Cambridge, MA 02138, USA. ${ }^{2}$ Smithsonian Astrophysics Observatory, 60 Garden Street, Cambridge, MA 02138, USA.

\section{Acknowledgements}

The travel expense for the symposium was supported by NASA Grant NNX12AG65G. We thank Nicole D. Melso for performing simulations to estimate the effective area and grasp for various telescope configurations. We also thank Martin Elvis for the useful comments.

\section{Competing interests}

The authors declare that they have no competing interests.

Received: 30 April 2015 Accepted: 7 January 2016

Published online: 29 February 2016

\section{References}

Altwegg K et al (2015) 67P/Churyumov-Gerasimenko, a Jupiter family comet with a high D/H ratio. Science 347:1261952. doi:10.1126/science.1261952 Binzel RP et al (2010) Earth encounters as the origin of fresh surfaces on nearEarth asteroids. Nature 463:331-334
Conconi P et al (2010) A wide field X-ray telescope for astronomical survey purposes: from theory to practice. Monthly notices of the Royal Astronomical Society 405:877-886

Ezoe $Y$ et al (2013) JUXTA: a new probe of X-ray emission from the Jupiter system. Adv in Space Res 51:1605-1621

Fraser GW et al (2010) The mercury imaging X-ray spectrometer (MIXS) on bepicolombo. Planetary and Space Science 58:79-95

Gubarev M et al (2012) The Marshall Space Flight Center development of mirror modules for the ART-XC instrument aboard the Spectrum-RoentgenGamma mission. SPIE 8443:84431U

Harrison F et al (2013) The Nuclear Spectroscopic Telescope Array (NuSTAR) high-energy X-Ray Mission. Astrophys J 770:103-121

Hong J, Romaine S (2014) Miniature lightweight x-ray optics (MiXO) for solar system exploration. SPIE 91441:91441F

Inamdar KN et al (2014) Modeling the expected performance of the REgolith X-ray imaging spectrometer (REXIS). SPIE 9222:922207

Lauretta D, Team O-R (2012) An overview of the OSIRIS-REx asteroid sample return mission. Lunar and Planetary Institute Science Conference 43:2491-2492

Nittler LR et al (2004) Bulk element compositions of meteorites: a guide for interpreting remote-sensing geochemical measurements of planets and asteroids. Antarctic Meteorite Res 17:231-251

Nittler LR et al (2011) The major-element composition of mercury's surface from MESSENGER X-ray spectrometry. Science 333:1847-1850

Noguchi T et al (2011) Incipient space weathering observed on the surface of Itokawa dust particles. Science 333:1121-1125

Noguchi T et al (2014) Mineralogy of four Itokawa particles collected from the first touchdown site. Earth, Planets, and Space 66:124-133. doi:10.1186/1880-5981-66-124

Okada T et al (2006) X-ray fluorescence spectrometry of asteroid Itokawa by Hayabusa. Science 312:1338-1341

Romaine $S$ et al (2014) Development of light weight replicated $x$-ray optics, II. SPIE 9144:91441H

Tachibana S et al (2013) The sampling system of Hayabusa-2: improvements from the Hayabusa sampler. Lunar and Planetary Science Conference 44:1880-1881

Trombka Jl et al (2000) The elemental composition of asteroid 433 eros: results of the NEAR-Shoemaker X-ray spectrometer. Science 289:2101-2105

\section{Submit your manuscript to a SpringerOpen ${ }^{\circ}$ journal and benefit from:}

- Convenient online submission

- Rigorous peer review

- Immediate publication on acceptance

Open access: articles freely available online

- High visibility within the field

- Retaining the copyright to your article

Submit your next manuscript at springeropen.com 